\title{
Clinical Presentation and Management of Severe Acute Renal Failure in McArdle Disease
}

\author{
Majdi Hamadeh, MD; Khalil Nasrallah, MD; Zeinab Ajami, MD; Rahil Zeaiter, MD; \\ Layan Abbas, MD; Samih Hamadeh, BS; and Jawad Fares, MD, MSc
}

McArdle disease, also known as glycogen storage disease type $\mathrm{V}$, is an autosomal recessive disease due to the absence of myophosphorylase activity, leading to the complete disruption of glycogen breakdown in muscles. We present a rare case of a Caucasian male, aged 26 years, who developed rhabdomyolysis-induced acute renal failure and uremic encephalopathy. Neurological examination and histopathological studies supported the diagnosis of McArdle disease. The severity of his symptoms necessitated urgent hemodialysis, upon which the patient reported improvement in status. Acute renal failure in McArdle disease usually resolves with supportive treatment and maintenance of regular physical activity. Nevertheless, in more severe cases, intensive care with urgent hemodialysis may be needed.A multidisciplinary approach is necessary for the adequate management of similar cases.

Keywords: McArdle Disease; Glycogen storage disease; Rhabdomyolysis; Acute renal failure; Neurology

$\mathrm{I}$ n 1951, McArdle described a glycogen storage disease that presents mainly as a myopathy. ${ }^{1}$ McArdle disease is one of the most common glycogen storage diseases, affecting approximately 1 per 100,000 people. $^{2}$ Patients typically present in adolescence or early adulthood with myalgias, fatigue, poor endurance, muscle swelling, and fixed weakness. ${ }^{3}$ Rhabdomyolysis and myoglobinuria are common, with more than $50 \%$ of patients experiencing them at least once. ${ }^{3,4}$ Although complete recovery of renal function is expected after uncomplicated attacks, rhabdomyolysisinduced acute renal failure can occur in rare cases. ${ }^{3,4}$

\section{Case Report}

A previously healthy Caucasian male, aged 26 years, presented to the emergency department with sudden onset of upper and lower limb pain, two episodes of black-colored urine, and one episode of vomiting. Symptoms were associated with generalized myalgia and a dry cough. The patient, a non-smoker with no history of substance abuse, noted that for the past 4 years he became easily fatigable upon minimal exercise. No other symptoms were noted.

On physical examination, he was ill-looking, conscious, cooperative, and oriented. A maculopapular rash sparing the palms and soles was noted. In addition, he had anicteric sclera, decreased bilateral air entry, and bilateral costovertebral angle tenderness. Neurological examination revealed significant deltoid muscle weakness.

Pertinent laboratory findings included elevated creatinine, creatine phosphokinase (CPK), lactate dehydrogenase (LDH), uric acid, aspartate aminotransferase (AST), and alanine aminotransferase (ALT). In addition, the patient was hypokalemic (Table 1). Urinalysis revealed proteinuria (+),
Corresponding Author: Jawad Fares, MD, MSc, Department of Neurological Surgery, Feinberg School of Medicine, Northwestern University, Chicago, IL, USA, Email: jawad.fares@northwestern.edu
Received: November 5, 2020

Revised: February 20, 2021

Accepted: March 4, 2021

doi: $|0.3| 2|/ \mathrm{cmr} .202| .|64|$ 
Table 1. Pertinent Laboratory Test Values at presentation

\begin{tabular}{lll}
\hline Laboratory Test & Patient Value & Normal Levels \\
\hline Creatinine (mg/dL) & 6.71 & $0.51-0.95$ \\
CPK (U/L) & 11088 & $<200$ \\
LDH (U/L) & 2145 & $135-235$ \\
Uric Acid (mg/dL) & 12.4 & $2-7$ \\
Potassium (mmol/L) & 3.47 & $3.5-5.1$ \\
AST (U/L) & 600.8 & $8-45$ \\
ALT (U/L) & 337 & $5-40$ \\
$\mathrm{HCO}_{3}$ (meq/L) & 17.9 & $22-26$ \\
\hline
\end{tabular}

CPK, creatine phosphokinase; LDH, lactate dehydrogenase; AST, aspartate aminotransferase; ALT, alanine aminotransferase; $\mathrm{HCO}_{3}$, bicarbonate

hemoglobinuria $(+++)$, white blood cells (WBCs) (6-8), red blood cells (RBCs) (2-4), and granular casts $(+)$. Otherwise, creatine kinase-MB (CPK-MB), troponin, gamma-glutamyl transferase (GGT), alkaline phosphatase, bilirubin, pro-time (PT), partial thromboplastin time (P-TT), and complete blood count with differential (CBCD) were within normal ranges, and urine and blood cultures were negative.

An echocardiogram done at presentation was normal. Computed tomography (CT) of the chest showed mild bilateral pleural effusion and bilateral basal segment collapse, with no masses or lymphadenopathies. Abdominal-pelvic ultrasound showed an engorged and prominent left kidney, measuring $14.6 \mathrm{~cm}$, with heterogeneous and mildly increased echogenicity.

The patient was admitted for further workup and treatment. The next day, he developed uremic encephalopathy with a creatinine of $7.71 \mathrm{mg} / \mathrm{dL}$ and a CPK level of $89243 \mathrm{U} / \mathrm{L}$. In addition, the patient had an elevated erythrocyte sedimentation rate (ESR) $(56$, normal $<20)$ and $24 \mathrm{~h}$ urine protein $(208$, range $28-141 \mathrm{mg}$ ). Thus, urgent dialysis was initiated. The patient was given doxycycline, allopurinol, and steroids, as well as necessary other symptomatic treatments. Upon further laboratory examinations, serum autoimmune serology was negative, which ruled out polymyositis. Electromyography (EMG) examination of right limbs and nerve conduction studies were normal; however, needle examination showed early recruitment and increased proportion of lower amplitude short duration and polyphasic motor units, suggesting mild myopathic process without membrane irritability. A skeletal muscle biopsy and histopathological studies outlined a glycogen storage disorder denoted by glycogen filled subsarcolemmal vacuoles "subsarcolemmal blebs" within many scattered muscle fibers with an overall excess in glycogen stores (Figure 1). Abnormal reticular glycogen deposits were also observed within many myofibers. Histochemical assessment of chosen enzymes involved in various glycogen storage diseases showed a complete loss of my phosphorylase activity (Figure 2). A mild variation in myofibers size and shape, a large number of myofibers with internalized nuclei, and fast twitch (type II) fiber predominance represented other observed pathological features. These findings strongly supported the diagnosis of McArdle disease.

Over the course of 11 days, the patient became clinically and hemodynamically stable, with creatinine, CPK, and liver enzymes trending down. The patient was discharged with a creatinine level of $1.6 \mathrm{mg} / \mathrm{dL}$ and CPK of $3037 \mathrm{U} / \mathrm{L}$.

\section{Discussion}

McArdle disease is an autosomal recessive defect affecting the myophosphorylase gene (PYGM), located on chromosome 11q13, with more than 100 mutations identified. Almost all mutations result in the total absence of the functional enzyme, with some rare exceptions. ${ }^{2}$ Glycogen, which acts as the fuel for anaerobic glycogenolysis, is critical for normal oxidative metabolism that powers brief, vigorous exercise. ${ }^{5}$ In McArdle disease there is an absence of the functional enzyme muscle glycogen phosphorylase, thus glycogen cannot be broken down. This results in rhabdomyolysis due to the combination of cellular stress from glycogen buildup, downregulation of sodium-potassium pump activity, increase of sarcoplasmic calcium, oxidative stress, and purine nucleotide metabolism that decreases cellular integrity. ${ }^{2,3} \quad$ Factors predisposing to acute renal failure in

Figure 1. Histology of a skeletal muscle biopsy. (A) Modified Gomori's Trichome (GT) and (B) Periodic acid-Schiff (PAS) stains show features of glycogen metabolism disorder characterized by sarcolemmal vacuolated areas within many muscle fibers $\left({ }^{*}\right)$, representing subsarcolemmal glycogen blebs (black arrows). An overall excess in glycogen content was also observed upon PAS staining. Photomicrographs represent a 200-fold magnification of frozen sections. Scale bar: $25 \mu \mathrm{m}$. 

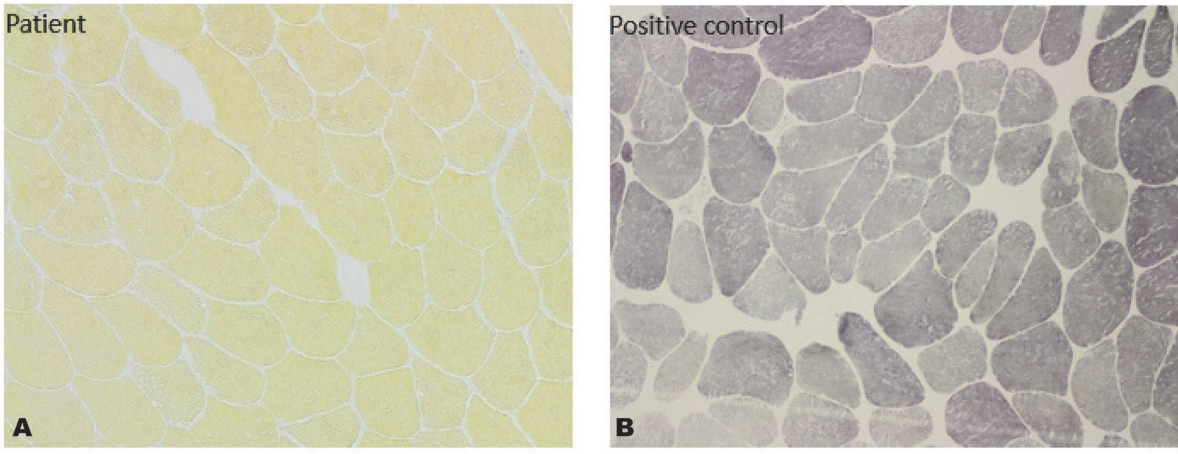

Figure 2. Hosto-enzymatic assessment of glycogen myophosphorylase activity. A complete loss of myophosphorylase activity was observed in the $(A)$ patient's muscle compared to $(\mathrm{B})$ positive control. Photomicrographs represent a 100-fold magnification of frozen sections.

McArdle disease include the severity of rhabdomyolysis, delayed supportive therapy, drug toxicity, and/or substance abuse. $^{6}$

McArdle disease is more common in the pediatric and young adolescent populations; although, rare cases in adults have been reported. In one case, a man, aged 57 years, with long-term idiopathic hypercreatine phosphokinasemia developed severe rhabdomyolysis and acute kidney injury after contracting an upper respiratory tract infection. A molecular diagnosis of McArdle disease was subsequently made. ${ }^{7}$ In two cases, men presented with muscular weakness, myalgia, and dark urine after almost drowning. ${ }^{8,9}$ Laboratory data showed severe rhabdomyolysis and necessitated hemodialysis. In our case, the patient was previously healthy. The diagnosis of McArdle disease required a histological analysis of a skeletal muscle biopsy. Definitive diagnosis can be made by performing a biochemical assay on the biopsy, which results in a negative phosphorylase reaction. ${ }^{10}$

Our patient necessitated urgent hemodialysis to reverse symptoms of severe acute renal failure and uremic encephalopathy. The mainstay of treatment involves a supervised exercise program and a high-complex carbohydrate, low fat diet to ensure constant supply of blood sugar, thus offering muscle protection during daily physical activity. ${ }^{11}$ Treatment for severe bouts of rhabdomyolysis needs timely supportive care, and can require intensive care admission, forced diuresis, or urgent renal dialysis. ${ }^{4}$

\section{Conclusion}

Despite its rarity, McArdle disease should be considered in patients who present with severe acute renal failure. Irregular symptomatology can be present long before seeking medical attention, which emphasizes the need for awareness at public health levels and among members of the medical community.
3. Merrit JL. Myophosphorylase deficiency (glycogen storage disease V, McArdle disease). UptoDate. Updated: June 29, 2020. Available at: https://www. uptodate.com/contents/myophosphorylase-deficiencyglycogen-storage-disease-v-mcardle-disease. Accessed on 28 October 2020.

4. Dimaur S, Andreu AL, Bruno C, Hadjigeorgiou GM. Myophosphorylase deficiency (glycogenosis type $\mathrm{V}$ McArdle disease). Curr Mol Med. 2002;2(2):189-196.

5. Vissing J, Haller RG. The effect of oral sucrose on exercise tolerance in patients with McArdle's disease. $\mathrm{N}$ Engl J Med. 2003;349(26):2503-2509.

6. Torner S, Tinel C, Soltani Z, Rifle G, Mousson C. Rhabdomyolysis with acute renal failure requiring dialysis in McArdle disease: A Role for the Antidepressant Venlafaxine? J Clin Psychopharmacol. 2016;36(4):406-408.

7. Satoh A, Hirashio S, Arima T, et al. Novel Asp511Thr mutation in McArdle disease with acute kidney injury caused by rhabdomyolysis. CEN Case Rep. 2019;8(3):194-199.

8. Pinto H, Teixeira AC, Oliveira N, Alves R. Wave of renal impairment. BMJ Case Rep. 2018;2018:bcr2017223437.

9. Zhao X, Li A, Soni M, Muriello MJ, Jones CH, Whittier WL. McArdle disease: a "pediatric" disorder presenting in an adult with acute kidney injury. CEN Case Rep. 2017;6(2):156-160.

10. Joshi PR, Deschauer M, Zierz S. McArdle disease: clinical, biochemical, histological and molecular genetic analysis of 60 patients. Biomedicines. 2020;8(2):33.

11. Nogales-Gadea G, Santalla A, Ballester-Lopez A, et al. Exercise and preexercise nutrition as treatment for McArdle disease. Med Sci Sports Exerc. 2016;48(4):673-9. 


\section{Author Affiliations}

Majdi Hamadeh, MD,*t; Khalil Nasrallah, MD,"t; Zeinab

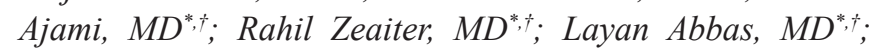
Samih Hamadeh, BS; Jawad Fares, MD, MSC

*Lebanese University Faculty of Medicine, Beirut, Lebanon ${ }^{*}$ Al-Zahraa Hospital University Medical Center, Beirut, Lebanon

University of Michigan-Dearborn, Dearborn, MI, USA

${ }^{\S}$ Department of Neurological Surgery, Feinberg School of Medicine, Northwestern University, Chicago, IL, USA 